\title{
In Situ Ellipsometric Study on Corrosion of Magnesium Alloys
}

\author{
Lingjie $\mathrm{LI}^{1}$, Jinglei LEI ${ }^{1}$ and Fusheng PAN ${ }^{2}$ \\ ${ }^{1}$ Coll. Chem. \& Chem. Eng., Chongqing University \\ ${ }^{2}$ Coll. Mat. Sci. E Eng., Chongqing University \\ P. R. China
}

\section{Introduction}

Magnesium is the $8^{\text {th }}$ most abundant element on the earth. Moreover, it is the lightest structural metal. Magnesium and alloys have numerous advantageous properties such as high specific strength, high thermal conductivity, high dimensional stability, high damping capacity, good machinability, good electromagnetic shielding characteristics and easilyrecycling characteristics. These excellent properties make them an ideal choice for a number of applications including automobile parts, computer and mobile components, sporting goods, aerospace equipment, household equipment, and implanted materials (Zhang \& Zhang, 2004).

However, magnesium and alloys are chemically active (the standard potential of $\mathrm{Mg}^{2+} / \mathrm{Mg}$ is -2.356 Vvs. N.H.E. at $25^{\circ} \mathrm{C}$ (Bard \& Faulkner, 2001).). Hence, they are extremely susceptible to corrosion whether in wet atmosphere or acid, neutral and weak alkaline solutions. The poor corrosion resistance seriously hinders their widespread use in many applications. Hence, a lot of researches have been performed on the corrosion of magnesium and alloys (Song \& Atrens, 2003; Song, 2005; Song, 2006; Wei at al, 2007; Li et al, 2008a-c).

In the investigations on the corrosion of magnesium alloys, the hydrogen evolution and weight loss measurements, the electrochemical techniques such as polarization curves and electrochemical impedance spectroscopy (EIS), the corrosion morphology characterization techniques such as optical microscopy and scanning electron microscopy (SEM), the corrosion products identification methods such as X-ray diffraction (XRD), X-ray photoelectron spectroscopy (XPS) and energy dispersive spectroscopy (EDS), etc., are often used. These conventional methods can provide the information of corrosion rate, corrosion resistance, corrosion morphology, corrosion products composition (Song, 2006). However, they fail to provide the micro-dynamic information of the "magnesium alloy - corrosive medium" interfaces, which is critical for understanding the corrosion mechanism and hence developing anti-corrosive magnesium alloys. Moreover, these conventional investigations are always carried out in such a way producing much perturbation of the "magnesium alloy - corrosive medium" interfaces. Therefore, employing appropriate methods to clarify the micro-dynamic details of the "magnesium alloy - corrosive medium" interfaces with minimal electrochemical and physical perturbation is very necessary. 
The use of in situ ellipsometry to investigate the metal-solution interfaces in the corrosive medium in real-time and without perturbation is well established. Our group simultaneously used ellipsometry and electrochemical measurements (cyclic voltammetry and EIS) to investigate the corrosion behaviors of copper electrode in borax solution and in weak-alkaline medium with the aggressive ion $\mathrm{Cl}^{-}$(Lei et al, $2001 \mathrm{a}-\mathrm{b}$ ). M.V. Vinnichenko et al. employed in situ ellipsometry to investigate stainless steel corrosion behavior in buffered solutions with amino acids (Vinnichenko et al, 2003). R. Bogdanowicz et al. used in situ ellipsometry and cyclic voltammetry measurements for the investigation of oxide growth on copper in $0.1 \mathrm{M} \mathrm{NaOH}$ solution (Bogdanowicz et al, 2009). In these studies, the optical properties and thickness of the metal-solution interfaces or the surface films on the metals were obtained by deconvoluting the ellipsometric data based on certain models. Thus the micro-dynamic details of the corrosion interfaces were clarified.

In this chapter, we briefly introduce the ellipsometric theory and present some original experimental and interpretive studies on the corrosion behaviors of magnesium alloys in some typical corrosive mediums, such as the simulated sea water, the simulated acid rain and the simulated industrial cooling water, with the principal means of in situ spectroscopic ellipsometry. In addition, the inhibition of sodium molybdate (an environmental-friendly inhibitor) on the corrosion of magnesium alloy in the simulated industrial cooling water is identified and explained. It has to be pointed out that in this work the "magnesium alloy corrosive medium" interfaces are considered as homogeneous and hence are regarded as a single layer with the aim to simplify the heavy deconvolution task. By deconvoluting the dynamic spectroscopic ellipsometric data, the refractive indexes and thickness of the "magnesium alloy - corrosive medium" interfaces during the different corrosion processes are obtained. The corrosion mechanism of magnesium alloys in these different corrosive mediums is proposed. The influence factors on the corrosivity of the mediums are discussed. The use of the in situ spectroscopic ellipsometry technique enables detailed characterization of the evolution of the thickness and optical properties of the "magnesium alloy - corrosive medium" interfaces during the corrosion processes. These studies are extremely important for clarifying the corrosion mechanism and reconstructing the corrosive interface structure of magnesium alloys.

\section{Experimental and ellipsometry theoretical}

\subsection{Experimental}

Materials. - Among the various magnesium alloys, the Mg-Al-Zn system, which includes alloys containing 2-10\% Al, combined with minor additions of zinc and manganese, is the earliest commercially used and the most widely used (Zhang \& Zhang, 2004). Hence, in this work, a Mg-Al-Zn magnesium alloy, namely AZ40, is chosen to be investigated. The AZ40 magnesium alloy rod was manufactured by Chongqing Magnesium, Ltd, P. R. China. Table1 lists the chemical composition of the alloy.

\begin{tabular}{|c|c|c|c|c|c|c|c|c|}
\hline Elements & $\mathrm{Al}$ & $\mathrm{Zn}$ & $\mathrm{Mn}$ & $\mathrm{Si}$ & $\mathrm{Cu}$ & $\mathrm{Ni}$ & $\mathrm{Fe}$ & $\mathrm{Mg}$ \\
\hline Content (mass \%) & 4.07 & 0.31 & 0.12 & 0.01 & 0.02 & 0.003 & 0.01 & Bal. \\
\hline
\end{tabular}

Table 1. Chemical composition of the AZ40 magnesium alloy. 
The AZ40 magnesium alloy electrode was cut from the rod of the alloy with cross-section area of $1.0 \mathrm{~cm}^{2}$ and embedded in epoxy resin holders. Before each experiment, the electrode surface was mechanically polished with $300,600,1000,1800 \#$ grit emery papers to get a plane mirror finish, then degreased ultrasonically in acetone for $3 \mathrm{~min}$, rinsed with distilled water and dried in air.

Corrosive mediums. - The corrosion resistance of one magnesium alloy is dependent on the medium to which it is exposed (Papavinasam, 2000). In this work, three typical corrosive mediums, including the simulated sea water, the simulated acid rain and the simulated industrial cooling water, are chosen to be investigated.

The simulated sea water is actually $3.5 \% \mathrm{NaCl}$ solution $(\mathrm{pH}=5.30)$, which is the most typical corrosive medium and represents the strong aggressive $\mathrm{Cl}-$ environment.

The acid rain is a serious pollution all over the world, which has attracted a lot of attention from environmentalists and researchers. Chongqing, as an important city of the southwest China with rapid developing of the magnesium industries, was one of the most serious acid rain areas in the world several years ago. In this work, the simulated acid rain is chosen as a typical corrosive medium for investigation. The simulated acid rain was prepared according to the research results on Chongqing rain water (Tang \& Bai, 2000). The composition of the Chongqing rain water is listed in Table2. The $\mathrm{pH}$ value of the simulated acid rain was adjusted to 4.67 (very close to the $\mathrm{pH}$ value of the rain water) by dilute sulphuric acid.

\begin{tabular}{|c|c|c|c|c|c|c|c|c|c|}
\hline Ions & $\mathrm{SO}_{4}^{2-}$ & $\mathrm{Cl}^{-}$ & $\mathrm{NO}_{3-}^{-}$ & $\mathrm{Ca}^{2+}$ & $\mathrm{Mg}^{2+}$ & $\mathrm{K}^{+}$ & $\mathrm{Na}^{+}$ & $\mathrm{NH}_{4}^{+}$ & $\mathrm{F}^{-}$ \\
\hline Content (mg/L) & 46.3 & 2.46 & 4.04 & 15.29 & 0.92 & 0.84 & 0.76 & 3.86 & 0.64 \\
\hline
\end{tabular}

Table 2. Composition of the Chongqing rain water (Tang \& Bai, 2000).

The industrial cooling water is an important medium in industrial production, which is corrosive to the metal equipments. The concentration of $\mathrm{Cl}$ - in the industrial cooling water is about $200 \mathrm{mg} / \mathrm{L}$, which is much lower than that in the sea water. In this work, the simulated industrial cooling water is regarded as a typical corrosive medium for study. Since our purpose focuses on the corrosion of AZ40 magnesium alloy caused by the aggressive ion $\mathrm{Cl}^{-}$ in the simulated cooling water, the simulated cooling water is actually $200 \mathrm{mg} / \mathrm{L} \mathrm{NaCl}$ solution $(\mathrm{pH}=5.23)$. Moreover, the inhibition of sodium molybdate, an environmentalfriendly inhibitor, on the corrosion of magnesium alloy in the simulated cooling water is studied. According to our previous work ( $\mathrm{Li}$ et al, $2008 \mathrm{~b}$ ), the concentration of sodium molybdate is chosen as $1000 \mathrm{mg} / \mathrm{L}$.

The corrosive mediums mentioned above were prepared from analytical grade chemicals and distilled water. All chemicals were offered by the Sinopharm Chemical Reagent Company of China.

Measurements. - Open circuit potential (OCP) and in situ spectroscopic ellipsometry measurements were conducted simultaneously at the room temperature.

OCP measurements were carried out on a CHI660B electrochemical workstation supplied by the Shanghai Chenhua Instruments Company of China. The electrochemical cell consisted of a conventional three-electrode configuration with a platinum sheet as the counter electrode and a saturated calomel electrode as the reference electrode. The working electrode was the cleaned AZ40 magnesium alloy electrode.

In situ spectroscopic ellipsometry measurements were performed using a Woollam ellipsometer M-2000U which was made in USA. Two ellipsometric parameters, $\Delta$ and $\psi$, 
were acquired. The measured wavelength range was from $245 \mathrm{~nm}$ to $1000 \mathrm{~nm}$ and the incident angle was fixed at $70^{\circ}$. The light beam reflected from the sample surface became weak due to the increasing roughness of the corrosion interfaces, which led to some spectral noise at 245-390 $\mathrm{nm}$ and 780-1000 $\mathrm{nm}$ during the measurements. Hence, only the experimental spectra within 390-780 nm were deconvoluted and reported in this work. A Woollam program (based on a Marquardt-Levenberg algorithm), WVASE32, was used for numerical analysis of the experimental ellipsometric data.

The refractive indexes of the corrosive mediums (the simulated sea water, the simulated acid rain, and the simulated industrial cooling water without and with sodium molybdate) were respectively 1.3391, 1.3323, 1.3324 and 1.3328, which were measured using an Abbé refractometer at the room temperature.

\subsection{Ellipsometry theoretical}

The following theoretical background for ellipsometry was described elsewhere (Bogdanowicz et al, 2009), which is excerpted here to help comprehending the ellipsometry technique.

The ellipsometry is an optical method for thin layer analysis. It is based on polarization state measurement of the light beam reflected from the sample. Such a procedure is used for thickness monitoring of dielectric and semiconductor layers synthesized during physical vapor deposition or chemical vapor deposition processes.

Following assumptions describing the analyzed optical model should be made. A model of a single layer for thin oxide film is considered (Stein et al, 1998). Linearly polarized light beam is incident on the sample at $\phi_{0}=70^{\circ}$ angle, where layer thickness is $d_{1}$. $N_{0}$ is a refractive index of medium, where substrate is located. Thin oxide films cover the strongly absorbing metal substrate characterized by refractive index $N_{2}$. The complex refractive indexes of investigated layers are denoted as $N_{1}$. For ellipsometric purposes, the complex-amplitude parameter $\rho$ is defined as a ratio of total reflection coefficients:

$$
\rho=\frac{r^{p}}{r^{s}}=\tan \Psi e^{j \Delta}
$$

The ellipsometric angles $\Delta$ and $\Psi$ represent the amplitude and phase ratio difference between $p$ - and s-polarization of light beam (Fujiwara, 2007). The amplitude reflectance coefficients in both polarization modes are expressed as follow:

$$
\frac{r^{p}}{r^{s}}=\left[\frac{r_{01}^{p}+r_{12}^{p} \exp \left(-j 2 \beta_{1}\right)}{1+r_{01}^{p} r_{12}^{p} \exp \left(-j 2 \beta_{1}\right)}\right] /\left[\frac{r_{01}^{s}+r_{12}^{s} \exp \left(-j 2 \beta_{1}\right)}{1+r_{01}^{s} r_{12}^{s} \exp \left(-j 2 \beta_{1}\right)}\right]
$$

where $r_{01}, r_{12}$ are the Fresnel reflection coefficient defined at boundaries of various $i$ medium, which depends on $N_{i}=n_{i}-i k_{i}$ complex refractive index (Fujiwara, 2007). The phase shift due to light beam refraction of angle $\phi_{1}$ is expressed by:

$$
\beta_{i}=4 \pi\left(\frac{d_{i}}{\lambda}\right) N_{i} \cos \phi_{i}
$$

All refractive indices $N_{0}, N_{1}$, and $N_{2}$ are complex functions of the $\lambda$ wavelength of incident light beam. The substitution of Fresnel reflection coefficients and Eqs. 2 and 3 in Eq. 1 gives 
complex ratio $\rho$ as a function of medium refractive indexes, light wavelength, and layer thickness.

\section{Results and discussion}

\subsection{AZ40 magnesium alloy in the air}

First, the experimental ellipsometric spectra of the AZ40 magnesium alloy in the air was collected and deconvoluted with the aim to determine the optical constants of the AZ40 magnesium alloy.

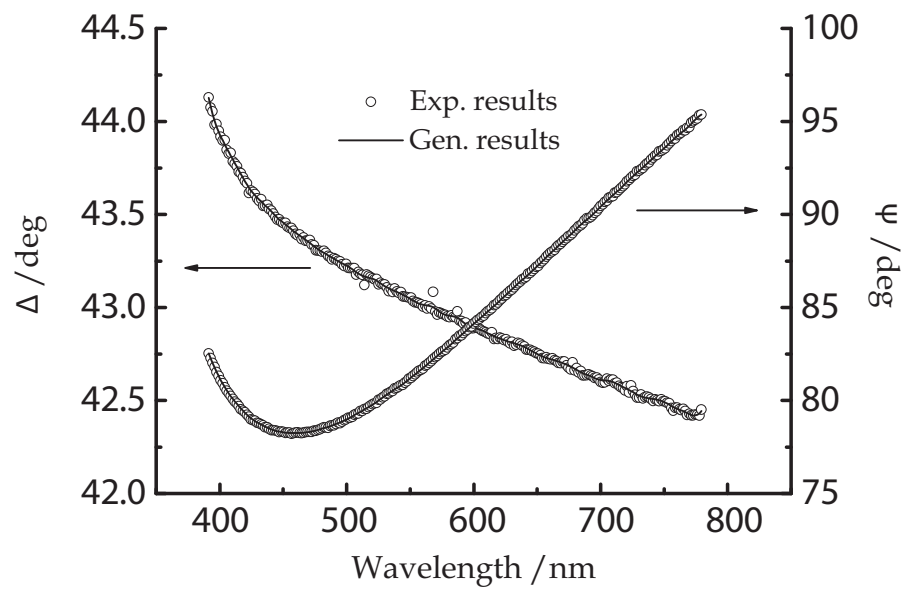

Fig. 1. Ellipsometric spectra of the AZ40 magnesium alloy in the air.

\begin{tabular}{|c|}
\hline Ambient-Air \\
\hline Native (hydr)oxide film \\
\hline AZ40 magnesium alloy \\
\hline
\end{tabular}

Fig. 2. Optical model for deconvoluting the ellipsometric spectra of the AZ40 magnesium alloy in the air.

Fig.1 shows the experimental ellipsometric spectra (as dots) of the AZ40 magnesium alloy in the air. Considering that a native (hydr)oxide film always exists on the alloy surface, a onelayer optical model (AZ40 magnesium alloy - native (hydr)oxide film - air) shown in Fig.2 is used to deconvolute the ellipsometric data. The generated ellipsometric spectra are shown as the solid lines in Fig.1, which are in good agreements with the experimental spectra. By 
deconvolution, the thickness of the native (hydr)oxide film is determined to be approximately $40.0 \mathrm{~nm}$. Moreover, the optical constants $(n, k)$ at different wavelengths for the AZ40 magnesium alloy are obtained, which are illustrated in Fig.3. For comparison, the optical constants $(n, k)$ (within 550-850 nm wavelength) of the AZ31 magnesium alloy obtained in our previous work ( $\mathrm{Li}$ et al, $2008 \mathrm{a}$ ) are also shown in Fig.3. The $n$ and $k$ values of the AZ40 magnesium alloy are larger than those of the AZ31 magnesium alloy at the same wavelength, which is attributed to the different surface status that caused by different alloy composition and pretreatments. However, the variation tendency of the $n$ and $k$ values with the wavelength is similar for the two magnesium alloys. These optical constants for different magnesium alloys can certainly enrich the current database for the optical constants of materials.

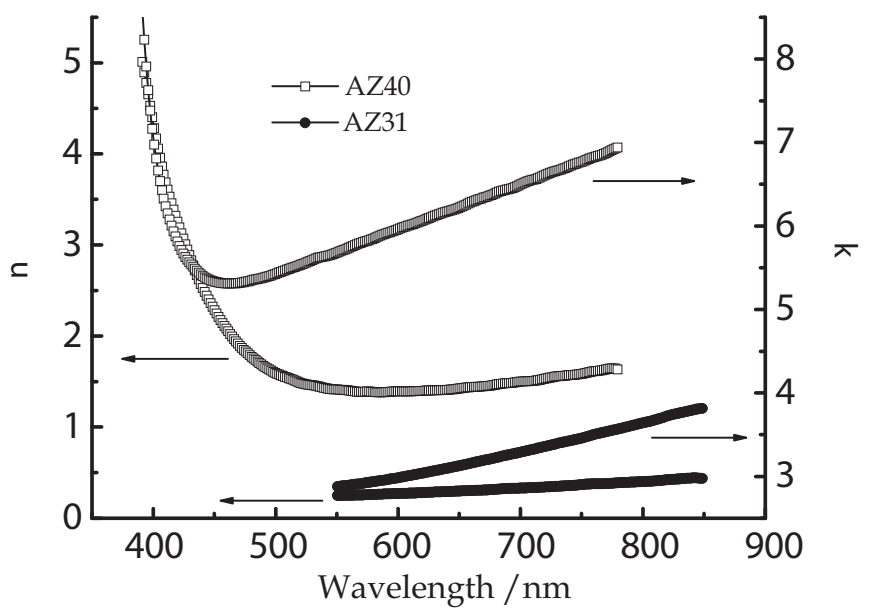

Fig. 3. Optical constants of the AZ40 and AZ31 magnesium alloys.

\subsection{AZ40 magnesium alloy in the simulated sea water}

The aggressive ion $\mathrm{Cl}^{-}$in the simulated sea water is strongly corrosive to most metals and alloys. Fig.4(a) illustrates the evolution of OCP of the AZ40 magnesium alloy upon immersion in the simulated sea water.

During the initial $4.8 \mathrm{~min}$, the OCP shifts positively from the beginning $-1.624 \mathrm{~V}$ to $-1.558 \mathrm{~V}$, which indicates that some corrosion products are unsolvable and stack on the sample surface to provide slight protection to the alloy. After that, the OCP drops suddenly to $1.571 \mathrm{~V}$ at $4.9 \mathrm{~min}$ and then gradually stabilizes at about $-1.588 \mathrm{~V}$ from $8.4 \mathrm{~min}$ till the immersion end. The sudden drop of the OCP is likely due to the falling off of the corrosion products from the alloy surface. The stable OCP reflects that the corrosion achieves dynamic-equilibrium and the corrosion rate varies little.

Fig.4(b) shows the corresponding variation of the ellipsometric parameters $\Delta$ and $\Psi$ (at the wavelength $\lambda=670.0 \mathrm{~nm}$ ) with the immersion time. The two parameters both increase slightly upon immersion.

To get the quantitative information on the thickness and optical properties of the corrosion interface during immersion, an optical model should be built and the dynamic spectroscopic ellipsometric data will be further deconvoluted. 
Generally, the corrosion interface is composition-complex and heterogeneous, which is very difficult to simulate. In the present work, to simplify the deconvolution, the corrosion interface is equivalent to a homogeneous layer. Hence, a one-layer model (AZ40 magnesium alloy - corrosion interface - corrosive medium) shown in Fig.5 is used to deconvolute the dynamic spectroscopic ellipsometric data during immersion. Some ellipsometric spectra generated by the deconvolution are shown as the solid lines in Fig.6, which are in good agreements with the experimental spectra shown as the dots in Fig.6.

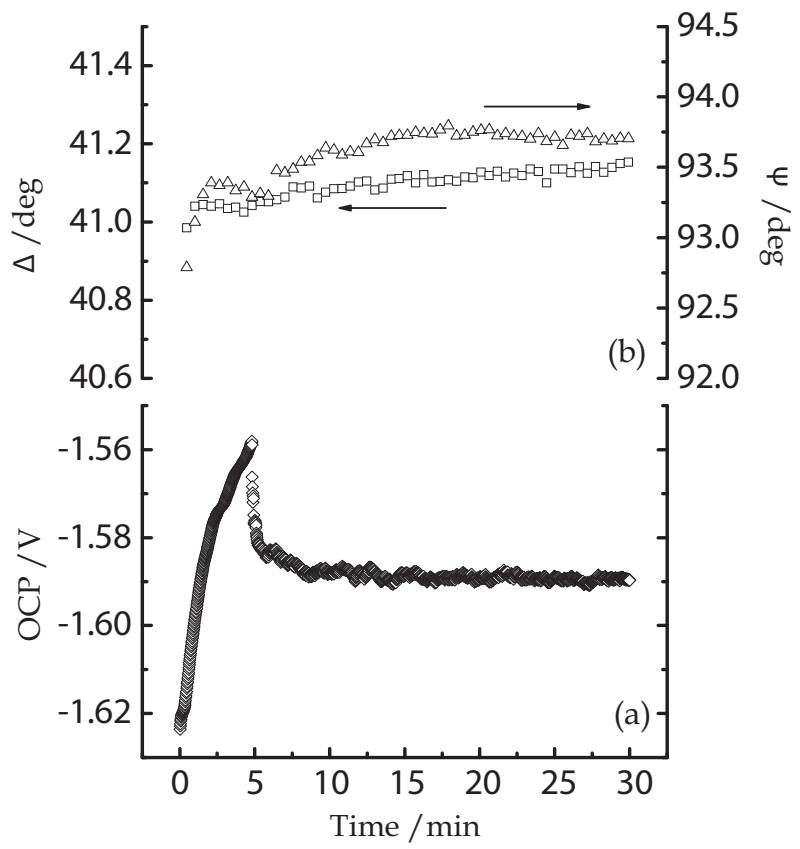

Fig. 4. Evolution of (a) OCP, (b) $\Delta$ and $\Psi$ (at $\lambda=670.0 \mathrm{~nm}$ ) upon immersion of the AZ40 magnesium alloy in the simulated sea water.

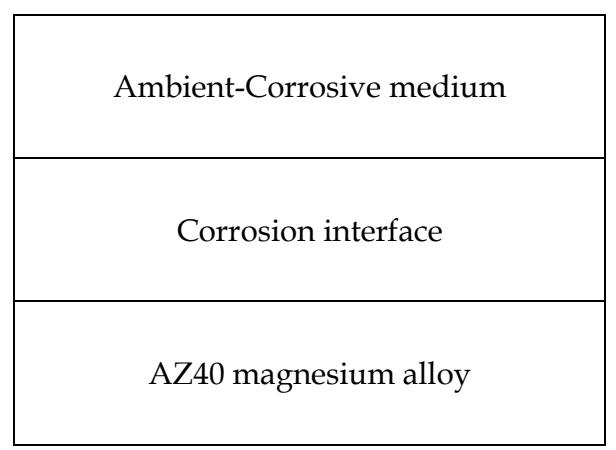

Fig. 5. Optical model for deconvoluting the ellipsometric spectra of the AZ40 magnesium alloy in the corrosive medium. 
Fig.7(a) and (b) respectively show the changes of the thickness $d$ and the refractive index $n$ (at $\lambda=670.0 \mathrm{~nm}$ ) of the corrosion interface layer with the immersion time. The variation of the thickness $d$ and the refractive index $n$ of the layer generally are correlated with the layer properties (Ord \& Huang, 1985). The decrease of the thickness $d$ usually corresponds to the dissolution of the surface film while the increase of the thickness $d$ often reflects the formation of the adsorption/precipitation film. The linear increase of the layer thickness $d$ with time indicates the poor protection performance of the layer. The changes of the layer thickness $d$ upon immersion follow a logarithmic function, which reflects that the layer can provide good protection. In addition, the larger refractive index $n$ often means the higher density and better protection of the layer. These rules are useful for the subsequent analyzing of the corrosion interface properties.
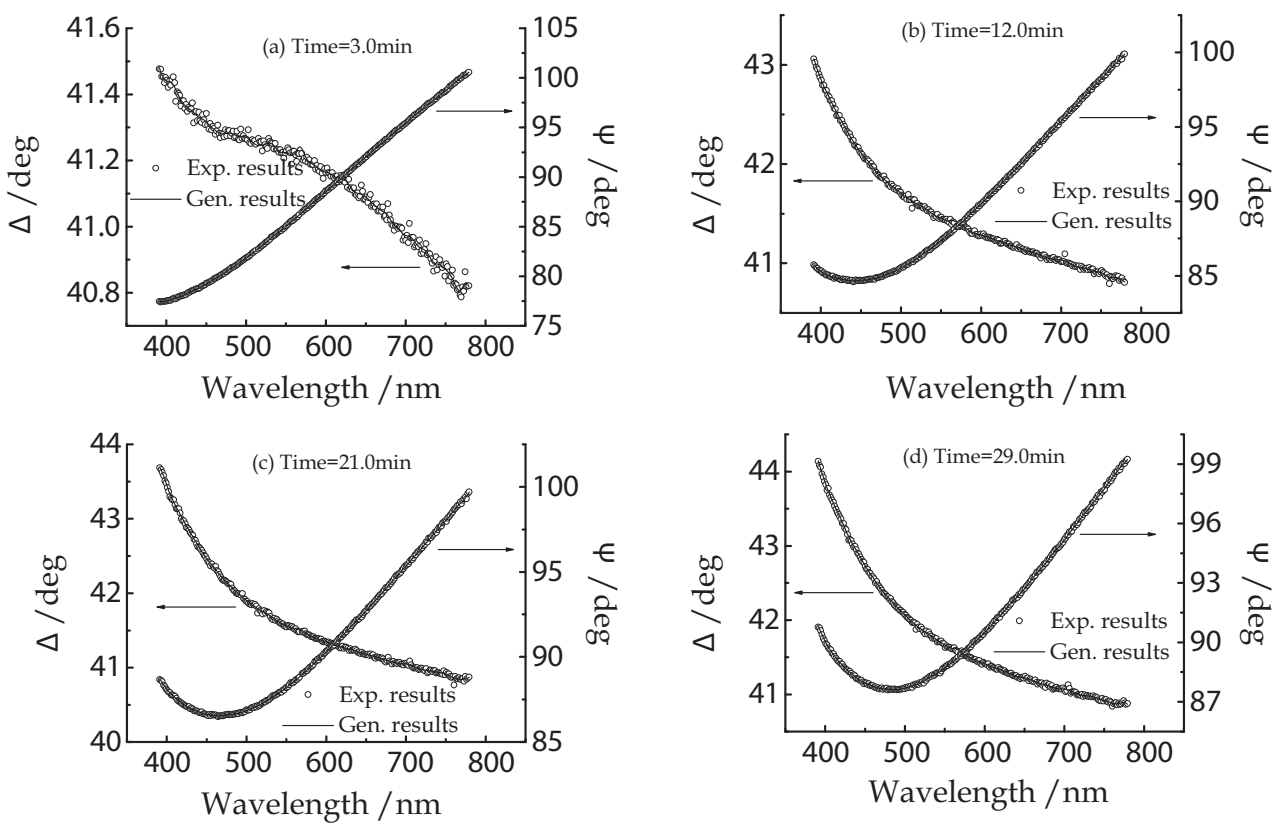

Fig. 6. Experimental and generated ellipsometric spectra during immersion of the AZ40 magnesium alloy in the simulated sea water for different time.

According to the evolution of the thickness $d$ and refractive index $n$ of the corrosion interface layer between the AZ40 magnesium alloy and the simulated sea water shown in Fig.7, and the evolution of OCP shown in Fig.4(a) as well as the SEM and EDS analysis in our previous work ( $\mathrm{Li}$ et al, $2008 \mathrm{c}$ ), the corrosion details of the AZ40 magnesium alloy in the simulated sea water can be speculated as follows.

The corrosion process can be divided into I, II and III three stages, as shown in Fig.7.

Stage I: It may include the dissolution of both the native (hydr)oxide film and the AZ40 magnesium alloy as well as the deposition of some unsolvable corrosion products on the AZ40 magnesium alloy surface, which can be inferred from the huge changes of the thickness $d$ and refractive index $n$ of the surface layer in the air and the first measured value (about $0.9 \mathrm{~min}$ ) after the alloy contacts with the simulated sea water. The thickness $d$ of the 
surface layer changes from $40.0 \mathrm{~nm}$ in the air to $165.0 \mathrm{~nm}$ at $0.9 \mathrm{~min}$ after the alloy contacts with the corrosive medium. The refractive index $n$ of the surface layer changes from 1.7325 to 1.5535 . Due to the strong corrosive nature of the simulated sea water, the reactions in the stage take place very quickly.

Stage II: The stage lasts within 0.9-8.0min immersion, during which the thickness $d$ of the corrosion interface layer decreases slightly and the refractive index $n$ increases little. This stage mainly includes the dissolution of the AZ40 magnesium alloy and the falling off and re-filing in of the corrosion products on the alloy surface. The falling and re-filing of the corrosion products makes the slightly decreasing hetergenerity of the corrosion interface. The decrease of the thickness $d$ of the corrosion interface layer in this stage follows a linear relationship:

$$
d / \mathrm{nm}=-0.8596 \times t / \mathrm{min}+165.45\left(R^{2}=0.9964\right)
$$

Stage III: This stage starts at $8 \mathrm{~min}$ till $30 \mathrm{~min}$ immersion end. In this stage, the thickness $d$ and refractive index $n$ of the corrosion interface layer are almost constant, which reflects that the corrosion of the AZ40 magnesium alloy in the simulated sea water has achieved the dynamic-equilibrium and the corrosion rate varies little.

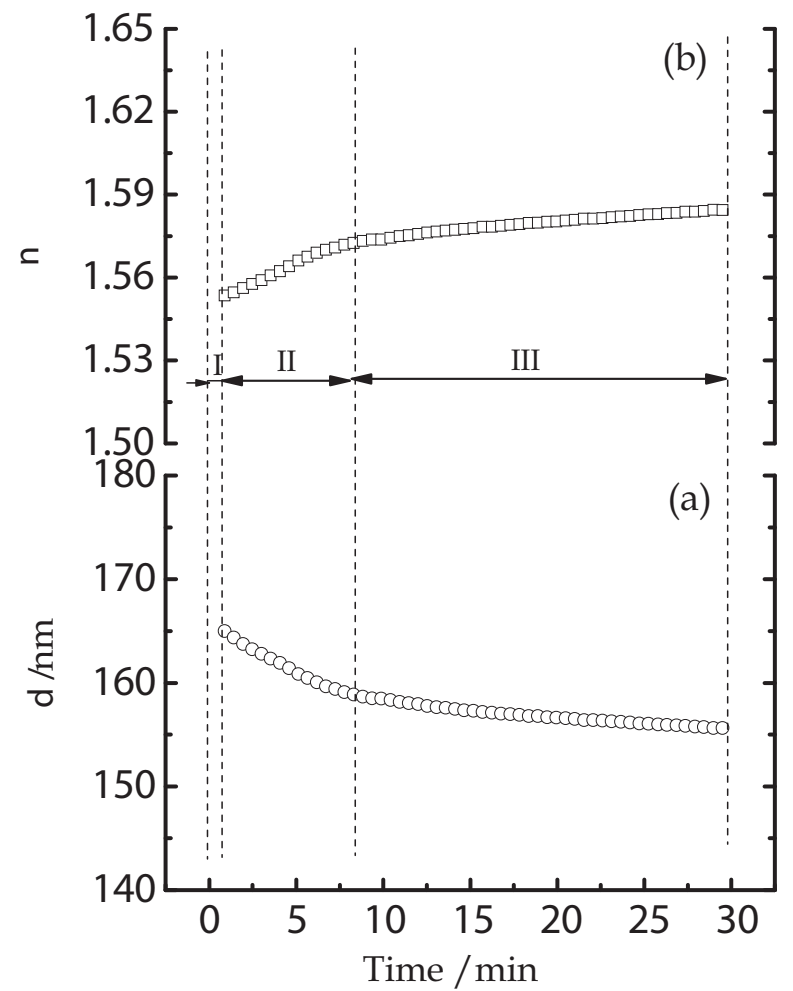

Fig. 7. Evolution of (a) the thickness $d$, (b) the refractive index $n$ (at $\lambda=670.0 \mathrm{~nm}$ ) of the corrosion interface between the AZ40 magnesium alloy and the simulated sea water during immersion. 


\subsection{AZ40 magnesium alloy in the simulated acid rain}

Fig.8(a) illustrates the evolution of OCP of the AZ40 magnesium alloy upon immersion in the simulated acid rain. The OCP shifts about $+100 \mathrm{mV}$ during the initial $4.8 \mathrm{~min}$ immersion and then keeps increasing slightly. Fig.8(b) shows the corresponding changes of the ellipsometric parameters $\Delta$ and $\Psi$ at the wavelength $\lambda=670.0 \mathrm{~nm}$ during immersion. The parameter $\Delta$ first increases slightly with immersion time, and then increases more obviously. The parameter $\Psi$ shows the trend of decreasing and then minor increasing. The quantitative information on the thickness and optical properties of the corrosion interface during immersion of the AZ40 magnesium alloy in the simulated acid rain is acquired by deconvoluting the dynamic spectroscopic ellipsometric data according to the one-layer model (AZ40 magnesium alloy - corrosion interface - corrosive medium) shown in Fig.5. Fig.9 illustrates some experimental (as dots) and generated ellipsometric spectra (as solid lines) during immersion, which are in good agreements.

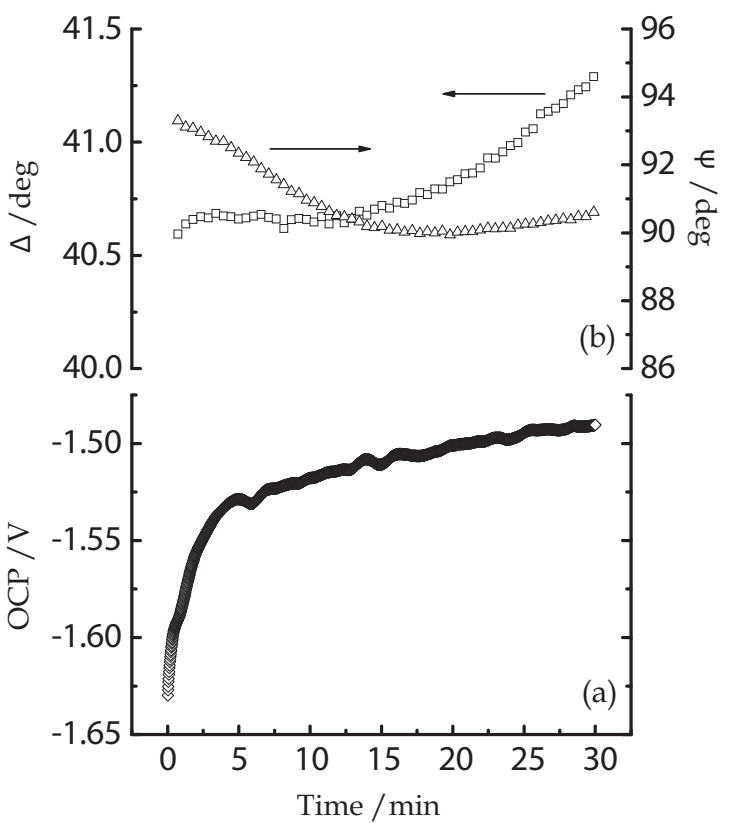

Fig. 8. Evolution of (a) OCP, (b) $\Delta$ and $\Psi$ (at $\lambda=670.0 \mathrm{~nm}$ ) upon immersion of the AZ40 magnesium alloy in the simulated acid rain.

Fig.10(a) and (b) respectively show the evolution of the thickness $d$ and the refractive index $n($ at $\lambda=670.0 \mathrm{~nm})$ of the corrosion interface layer upon immersion. Combining with the evolution of OCP shown in Fig.8(a) as well as the other electrochemical results in our previous study, the corrosion details of the AZ40 magnesium alloy in the simulated acid rain can be conferred.

Similarly, the corrosion evolution undergoes I, II and III three stages, which are illustrated in Fig.10.

Stage I: Due to the lower $\mathrm{pH}$ value of the simulated acid rain, the natural (hydr)oxide film on the AZ40 magnesium alloy surface dissolves very quickly after the alloy contacts with 
the simulated acid rain and then the alloy dissolves. From the large increase of the thickness $d(40.0 \mathrm{~nm}$ in the air and $148.4 \mathrm{~nm}$ at $0.8 \mathrm{~min}$ after the alloy contacts with the simulated acid rain) as well as the decrease of the refractive index $n$ (changing from 1.7325 to 1.6153) of the surface layer, it can be inferred that the anions such as $\mathrm{SO}_{4}{ }^{2-}$ and $\mathrm{NO}_{3}$ - in the simulated acid rain easily adsorb on the alloy surface and the unsolvable corrosion products likely precipitate on the surface to form the surface layer. The above dissolution, adsorption and precipitation occur very rapidly.
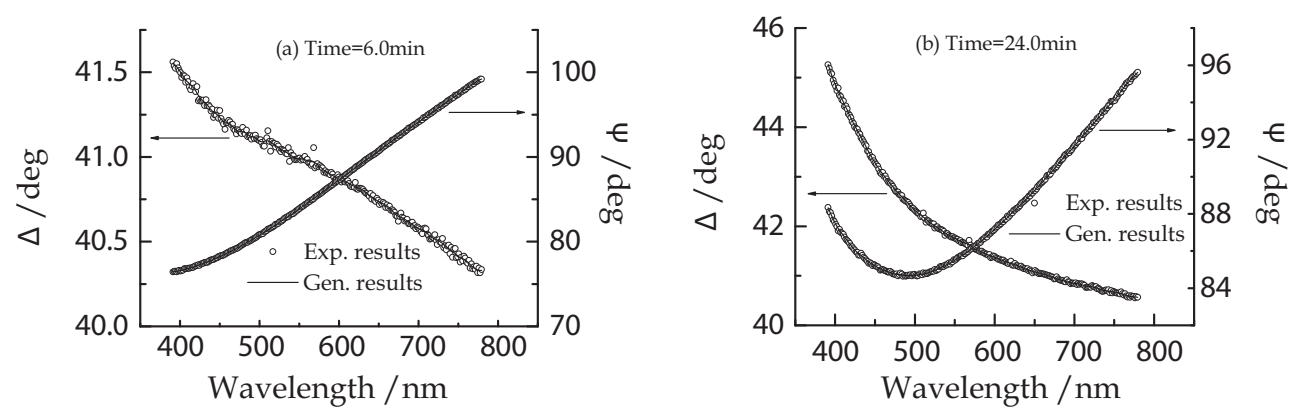

Fig. 9. Experimental and generated ellipsometric spectra during immersion of the AZ40 magnesium alloy in the simulated acid rain for different time.

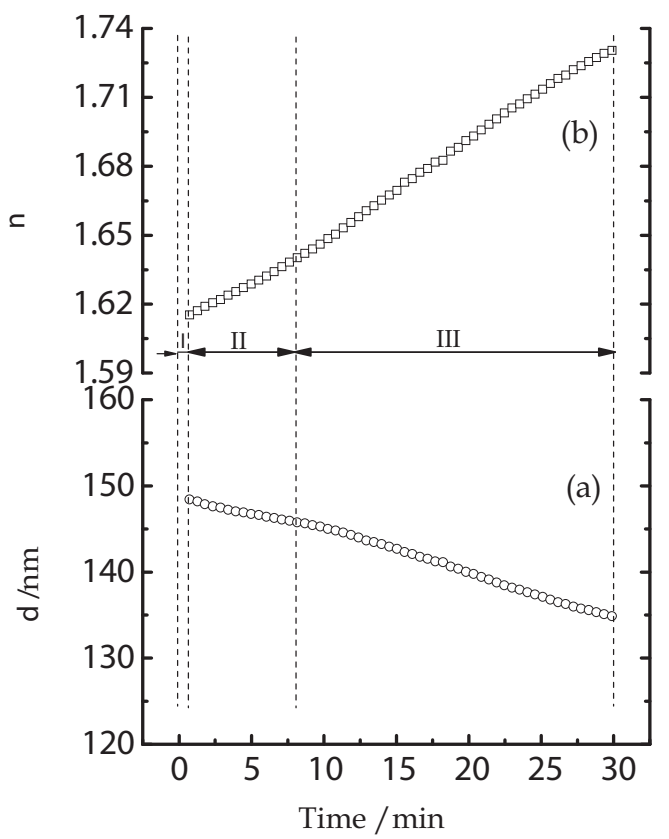

Fig. 10. Evolution of (a) the thickness $d$, (b) the refractive index $n$ (at $\lambda=670.0 \mathrm{~nm}$ ) of the corrosion interface between the AZ40 magnesium alloy and the simulated acid rain during immersion. 
Stage II: The stage lasts within 0.8-8.2min immersion of the AZ40 magnesium alloy in the simulated acid rain. At about $8.2 \mathrm{~min}$ immersion, there is a turning point on the $d$ (thickness)- $t$ (time) curve and $n$ (refractive index)- $t$ curve, after which the thickness $d$ decreases and the refractive index $n$ increases at a different rate. Hence, this stage is inferred to mainly include the dissolution of the AZ40 magnesium alloy, the desorption and readsorption of the anions as well as the falling off and re-filing in of the corrosion products on the alloy surface, which make the decreasing hetergenerity of the corrosion interface. The thickness $d$ of the corrosion interface layer decreases linearly as the following relationship:

$$
d / \mathrm{nm}=-0.3448 \times t / \mathrm{min}+148.50\left(R^{2}=0.9946\right)
$$

Stage III: This stage starts at $8.2 \mathrm{~min}$ till $30 \mathrm{~min}$ immersion end. The processes occur in this stage are similar to those in stage II. But the rates of the dissolution of the AZ40 magnesium alloy, of the desorption and re-adsorption of the anions as well as of the falling off and refiling in of the corrosion products on the alloy surface in these two stages are slightly different, which makes the different decrease rate of the thickness $d$ and the different increase rate of the refractive index $n$ of the corrosion interface layer in these two stages. The decrease of the thickness $d$ of the corrosion interface layer in this stage follows a linear relationship:

$$
d / \mathrm{nm}=-0.5314 \times t / \mathrm{min}+150.54\left(R^{2}=0.9994\right)
$$

\subsection{AZ40 magnesium alloy in the simulated cooling water}

Fig.11(a) shows the evolution of OCP of the AZ40 magnesium alloy upon immersion in the simulated cooling water without and with sodium molybdate (the inhibitor). The OCP in these two cases both shifts about $+120 \mathrm{mV}$ once the magnesium alloy contacts with the mediums, and then keeps constant. The OCP in simulated cooling water with the inhibitor is always more positive than that without the inhibitor. Fig.11(b) and (c) respectively show the corresponding changes of the ellipsometric parameters $\Delta$ and $\Psi$ ( at $\lambda=670.0 \mathrm{~nm}$ ) during immersion. The parameter $\Delta$ in the two cases both increases with time, which is more obvious in the simulated cooling water with the inhibitor. The parameter $\Psi$ shows the trend of increasing, decreasing, and then increasing with time in the simulated cooling water without the inhibitor, which is opposite to the trend in the presence of the inhibitor. By deconvoluting the dynamic spectroscopic ellipsometric data according to the one-layer model (AZ40 magnesium alloy - corrosion interface - corrosive medium) shown in Fig.5, the quantitative information on the thickness and optical properties of the corrosion interface during immersion can be obtained. Fig.12 and Fig.13 respectively show some experimental (as dots) and corresponding generated ellipsometric spectra (as solid lines) during immersion of the AZ40 magnesium alloy in the simulated cooling water without and with the inhibitor. The experimental spectra agree well with the generated ones.

Fig.14(a) and (a') respectively illustrate the thickness $d$ evolution of the corrosion interface layer upon immersion of the AZ40 magnesium alloy in the simulated cooling water without and with the inhibitor. Fig.14(b) and ( $\left.b^{\prime}\right)$ respectively show the corresponding changes of the refractive index $n$ (at $\lambda=670.0 \mathrm{~nm}$ ) of the corrosion interface layer. Combining with the evolution of OCP shown in Fig.11(a) as well as the EIS, SEM and EDS analysis in our previous research ( $\mathrm{Li}$ et al, $2008 \mathrm{~b}$ ), the corrosion details of the AZ40 magnesium alloy in the simulated cooling water without and with the inhibitor are presumed. 

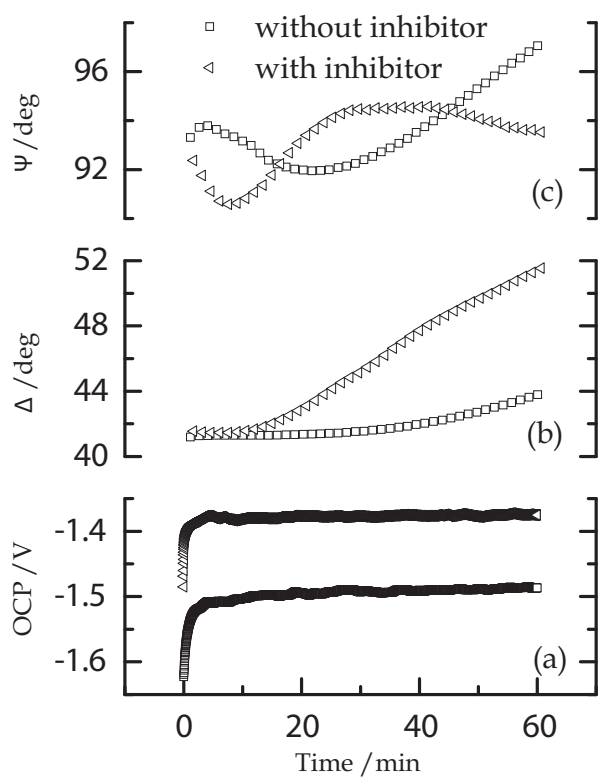

Fig. 11. Evolution of (a) OCP, (b) $\Delta$ and (c) $\Psi$ (at $\lambda=670.0 \mathrm{~nm}$ ) upon immersion of the AZ40 magnesium alloy in the simulated cooling water without and with the inhibitor.
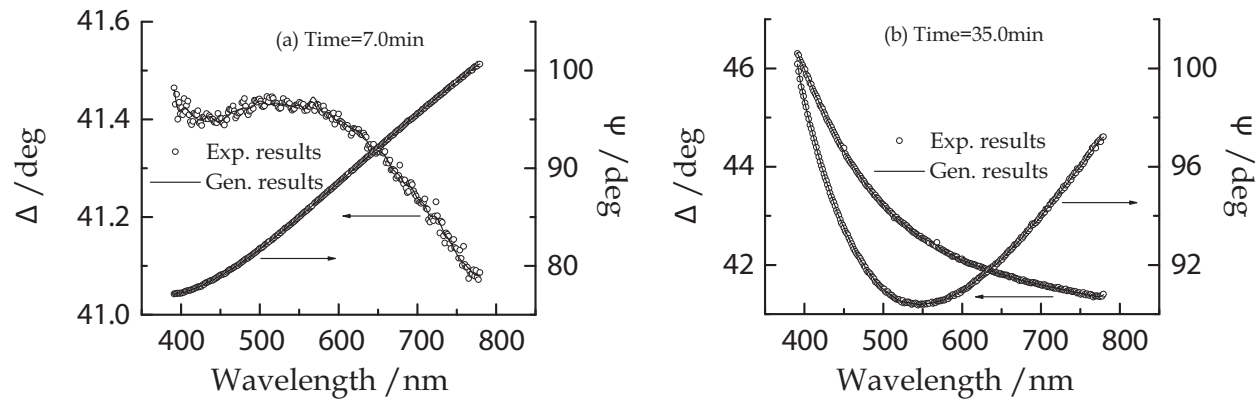

Fig. 12. Experimental and generated ellipsometric spectra during immersion of the AZ40 magnesium alloy in the simulated cooling water without the inhibitor.

As shown in Fig.14, the corrosion processes of the AZ40 magnesium alloy in the simulated cooling water without the inhibitor include I, II and III three stages.

Stage I: The stage lasts $4.0 \mathrm{~min}$ after the alloy contacts with the medium. The thickness $d$ and refractive index $n$ of the corrosion interface layer change very little during this stage, which indicates that the stage mainly corresponds to the dissolution of the native (hydr)oxide film on the alloy surface.

Stage II: The stage lasts within 4.0-19.0min immersion. The thickness $d$ and refractive index $n$ of the corrosion interface layer both increase slightly. This stage is speculated to mainly include the minor dissolution of the alloy and the adsorption of $\mathrm{OH}^{-}$that produced during the dissolution process to form the adsorption film on the alloy surface. 


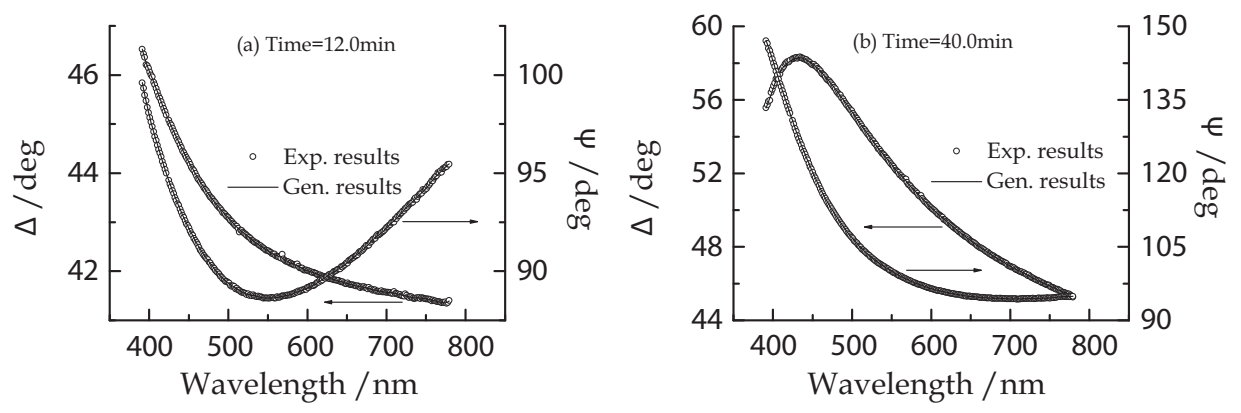

Fig. 13. Experimental and generated ellipsometric spectra during immersion of the AZ40 magnesium alloy in the simulated cooling water with the inhibitor.
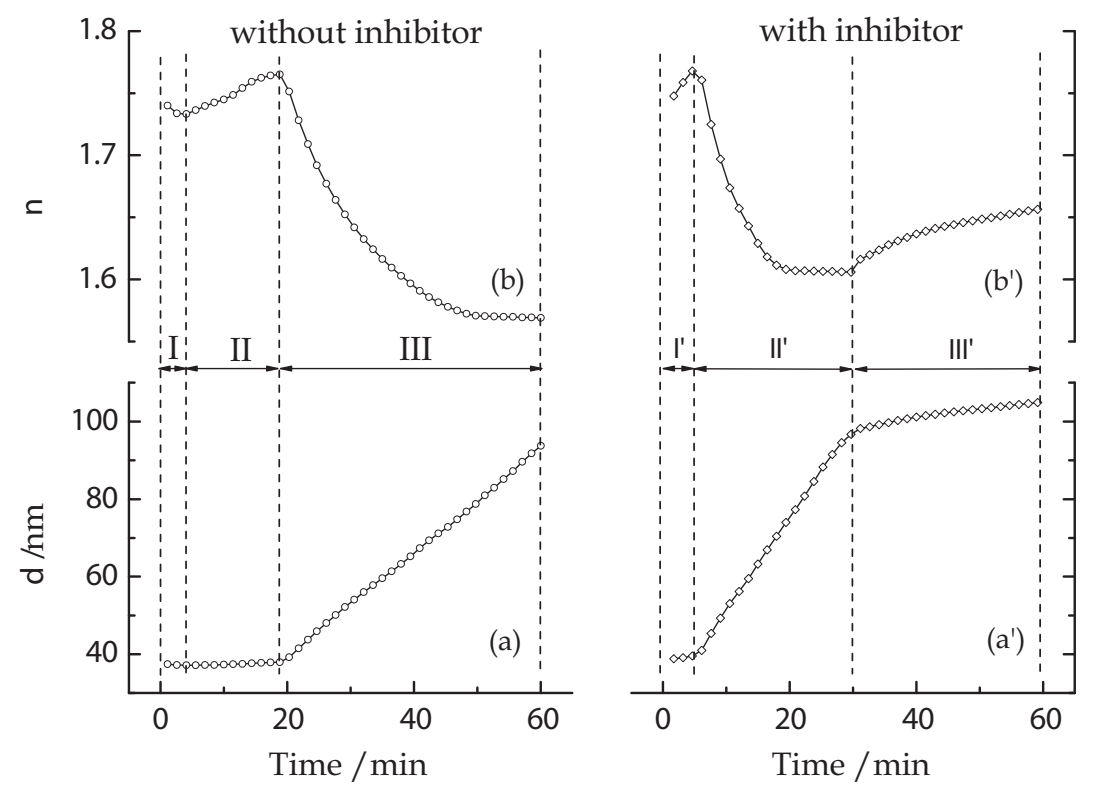

Fig. 14. Evolution of (a) and (a') the thickness $d,(b)$ and ( $\left.b^{\prime}\right)$ the refractive index $n$ (at $\lambda=670.0$ $\mathrm{nm}$ ) of the corrosion interfaces between the AZ40 magnesium alloy and the simulated cooling water without and with the inhibitor.

Stage III: This stage starts at $19.0 \mathrm{~min}$ till $60.0 \mathrm{~min}$ immersion end. The refractive index $n$ of the corrosion interface layer decreases. The layer thickness increases linearly with the immersion time, which can be described as the following relationship:

$$
d / \mathrm{nm}=1.3386 \times t / \mathrm{min}+12.631 \quad\left(R^{2}=0.9997\right)
$$

This stage is inferred to mainly correspond to the minor dissolution of the alloy and the deposition of the unsolvable corrosion products such as $\mathrm{Mg}(\mathrm{OH})_{2}$ on the alloy surface. Similarly, the corrosion processes of the AZ40 magnesium alloy in the simulated cooling water with the inhibitor can be divided into I', II' and III' three stages, as shown in Fig.14. 
Stage I': The stage lasts 5.0min after the alloy contacts with the medium. The thickness $d$ of the corrosion interface layer has little change while the refractive index $n$ increases slightly, which indicates that the stage mainly includes the slight dissolution of the native (hydr)oxide film, and the adsorption of the poly-molybdenum anions ( such as $\left[\mathrm{Mo}_{7} \mathrm{O}_{24}\right]^{6-}$ and $\left[\mathrm{Mo}_{8} \mathrm{O}_{26}\right]^{4-}$ ) that generated by the polymerization of the inhibitor ions (Mu et al, 2006) as well as $\mathrm{OH}^{-}$that produced during the dissolution process to form the adsorption film on the alloy surface.

Stage II': The stage lasts within 5.0-30.0min immersion. The refractive index $n$ of the interface layer decreases and gradually tends to be constant. The layer thickness $d$ increases linearly with the immersion time. The linear relationship can be described as:

$$
d / \mathrm{nm}=2.3786 \times t / \mathrm{min}+27.559\left(R^{2}=0.9998\right)
$$

This stage is speculated to mainly include the slight dissolution of the alloy, the precipitation of magnesium poly-molybdate and the deposition of the unsolvable corrosion products such as $\mathrm{Mg}(\mathrm{OH})_{2}$ on the alloy surface.

Stage III': This stage starts at $30.0 \mathrm{~min}$ till $60.0 \mathrm{~min}$ immersion end. The refractive index $n$ of the interface layer keeps stable increasing. The layer thickness increases logarithmic with the following relationship:

$$
d / \mathrm{nm}=81.605+6.1962 \times \ln (t / \min -16.802)\left(R^{2}=0.9995\right)
$$

This stage is presumed to mainly correspond to the increasing homogenerity and compactness of the interface layer, which makes the layer more protective to the alloy.

\subsection{Discussion of the influence of the corrosive medium}

As well known, $\mathrm{Mg}^{2+}$ is the sole stable magnesium species in aqueous solutions with $\mathrm{pH}$ value lower than 8.50 . Therefore, the native (hydr)oxide film on the AZ40 magnesium alloy surface will dissolve after the alloy contacts with the acidic corrosive medium especially the medium with low $\mathrm{pH}$ value such like the simulated acid rain in this work. After the dissolution of the (hydr)oxide film, the primary anodic reaction (the dissolution of magnesium) occurs:

$$
\mathrm{Mg} \rightarrow \mathrm{Mg}^{2+}+2 \mathrm{e}^{-}
$$

Spontaneously, the cathodic reaction (the reduction of protons) occurs:

$$
2 \mathrm{H}_{2} \mathrm{O}+2 \mathrm{e}^{-} \rightarrow 2 \mathrm{OH}^{-}+\mathrm{H}_{2}
$$

The $\mathrm{OH}^{-}$producing in reaction (11) leads to the $\mathrm{pH}$ increase at the corrosion interface. Once the interfacial $\mathrm{pH}$ is high enough, $\mathrm{Mg}^{2+}$ will precipitate as solid hydroxide $\mathrm{Mg}(\mathrm{OH})_{2}$ (The solubility product constant $\mathrm{p} K_{\mathrm{sp}}$ of $\mathrm{Mg}(\mathrm{OH})_{2}$ is 11.25 (Dean, 1999).) and then deposit on the alloy surface.

If some special ions exist in the corrosive medium, the unsolvable precipitates, such as magnesium poly-molybdate in the simulated cooling water with sodium molybdate, will deposit on the alloy surface, too.

From the analysis in sections 3.2-3.4, it is obvious that in the present work the simulated sea water is the most corrosive medium to the AZ40 magnesium alloy, and the simulated acid rain is secondly corrosive while the simulated cooling water is the least corrosive. The dissolution of the native (hydr)oxide film as well as the AZ40 magnesium alloy in the 
simulated sea water takes place very rapidly. The thickness of the corrosion interface layer between the AZ40 magnesium alloy and the simulated sea water changes linearly and the layer has smaller refractive index values, which indicates the poor protection of the layer to the alloy. In the simulated acid rain, the native (hydr) oxide film and the AZ40 magnesium alloy also dissolve very quickly due to the more acidic nature of the medium. The thickness of the corrosion interface layer changes linearly while the refractive indexes of the layer are not small, which indicates that the layer can provide some protection to the alloy and the corrosion of the alloy in the simulated acid rain is not very serious. In case of the simulated cooling water, the dissolution rate of the native (hydr)oxide film on the alloy surface is slow and the AZ40 magnesium alloy dissolves slightly. The thickness of the interface layer increases linearly and the layer has large refractive indexes, which indicates that the layer can provide good protection to the alloy and the corrosion of the alloy in the simulated cooling water is slight. With the addition of the inhibitor (sodium molybdate) in the simulated cooling water, the dissolution rate of the native (hydr)oxide film on the alloy surface is much slower. The thickness of the interface layer increases logarithmic and the layer has larger refractive indexes, which reflects that the layer can provide much stronger protection to the alloy.

Hence, the $\mathrm{pH}$ value of the corrosive medium and the concentration of the aggression ion $\mathrm{Cl}^{-}$in the medium greatly determine the corrosive nature of the medium. The lower $\mathrm{pH}$ value and the more $\mathrm{Cl}^{-}$ions, the more corrosive of the medium, and thus the more poor protection of the corrosion interface layer and the more serious corrosion of the alloy. The molybdate inhibits the corrosion of the AZ40 magnesium alloy in the simulated cooling water by improving the homogenerity and compactness of the interface between the alloy and the corrosive medium.

\section{Summary and outlook}

In this work, the feasibility of using in situ spectroscopic ellipsometry as a powerful tool for obtaining a better understanding of the corrosion details and mechanism of the AZ40 magnesium alloy in three typical corrosive mediums (the simulated sea water, the simulated acid rain, and the simulated industrial cooling water without or with sodium molybdate) was explored. The refractive indexes and thickness of the "magnesium alloy - corrosive medium" interfaces upon different corrosion evolution are obtained by deconvoluting the dynamic spectroscopic ellipsometric data according to a one-layer model. The corrosion details and mechanism of the AZ40 magnesium alloy in different corrosive mediums are analyzed by correlating the variation of the refractive indexes and thickness of the corrosion interfaces with the layer protective properties. The inhibition mechanism of sodium molybdate on the corrosion of the AZ40 magnesium alloy in the simulated cooling water is elucidated. The corrosion dynamics during different stages is explicit. The corrosivity of the three corrosive mediums in the present work is compared and the influence factors on the corrosivity of the medium are discussed.

The corrosion details and mechanism described in the present work are very useful to reconstruct the corrosive interface structure of magnesium alloys in the corrosive mediums and will absolutely supplement the current investigations on the corrosion of magnesium alloys by the conventional methods. Moreover, the optical constants of the AZ40 magnesium alloy especially the abundant optical constants of the "AZ40 magnesium alloy corrosive medium" interfaces upon different corrosion evolution obtained in the present 
work can greatly enrich current database for optical constants of materials. Thus, the application of in situ spectroscopic ellipsometry to the study of the corrosion processes for magnesium alloys shows considerable promise.

However, some work remains to be done to refine the interpretive approach. For example, adopting a multi-components EMA (Efficient medium approximation) model instead of a one-layer model to represent the corrosion interface during deconvoluting the dynamic spectroscopic ellipsometric data, the results are expected to better reflect the compositioncomplex and heterogeneous natures of the corrosion interface.

\section{Acknowledgments}

The authors thank the Natural Science Foundation of Chongqing government (CSTC 2009BA4023), the National Natural Science Foundation of China (NSFC 20803097, 20603049), the National Outstanding Youth Science Foundation of China (NSFC 50725413) and the Fundamental Research Funds for the Central Universities (CDJRC10220002) for financially supporting the research.

\section{References}

Bard, A. \& Fanlkner, L. (2001). Electrochemical methods, fundamentals and applications, John Wiley \& Sons, Inc., ISBN 0-471-04372-9, New York.

Bogdanowicz, R.; Ryl, J.; Darowicki, K.; et al. (2009). Ellipsometric study of oxide formation on Cu electrode in $0.1 \mathrm{M} \mathrm{NaOH}$. Journal of Solid State Electrochemistry, 13, 1639-1644, ISSN 1432-8488.

Dean, J. (1999). Lange's handbook of chemistry (15th Ed.), McGraw-Hill, Inc., ISBN 0-07-0163847, New York.

Fujiwara, H. (2007). Spectroscopic ellipsometry, principles and applications, John Wiley \& Sons Ltd., ISBN 978-0-470-01608-4, West Sussex.

Lei, J.; Li, L.; Zhang, S.; et al. (2001 a). Studies on corrosion behavior of copper electrode in weak alkaline solution. Acta Chimica Sinica, 59, 1216-1221, ISSN 0567-7351.

Lei, J.; Li, L.; Cai, S.; et al. (2001 b). Effect of $\mathrm{Cl}^{-}$on the corrosion behavior of copper electrode in weak-alkaline medium. Acta Physico-Chimica Sinica, 17, 1107-1111, ISSN 10006818.

Li, L.; Lei, J.; Yu, S.; et al. (2008 a). In situ ellipsometric studies of formation kinetics of rare earth metal conversion coating on magnesium alloys. Physica Status Solidi (c), 5: 1308-1311, ISSN 1610-1642.

Li, L.; Lei, J.; Yu, S.; et al. (2008 b). Inhibition of corrosion of magnesium alloy by molybdate in simulated cooling water. Journal of Chemical Industry and Engineering (China), 59: 1223-1227, ISSN 0438-1157.

Li, L.; Yu, S.; Lei, J.; et al. (2008 c). Corrosion behavior and mechanism of AZ40 magnesium alloy in simulated sea water. Journal of Chongqing University, 31, 702-706, ISSN 10052909.

Ord, J. \& Huang, Z. (1985). An optical study of the deposition, discharge, and recharge of manganese dioxide films. Journal of The Electrochemical Society, 132, 1183-1186, ISSN 0013-4651.

Papavinasam, S. (2000). Corrosion inhibitors, In: Uhlig's corrosion handbook (2nd Ed.), Ravie, R. (Ed.), 1089-1105, John Wiley \& Sons, Inc., ISBN 0-471-15777-5, New York. 
Song, G. \& Atrens, A. (2003). Understanding magnesium corrosion. Advanced Engineering Materials, 5, 837-858, ISSN 1616-301X.

Song, G. (2005). Recent progress in corrosion and protection of magnesium alloys. Advanced Engineering Materials, 7, 563-586, ISSN 1616-301X.

Song, G. (2006). The corrosion and protection of magnesium alloys, Chemical Industry Press of China, ISBN 7-5025-8565-6, Beijing.

Stein, N.; Johann, L.; Rapin, C.; et al. (1998). In situ ellipsometric study of copper passivation by copper heptanoate through electrochemical oxidation. Electrochimica Acta, 43, 3227-3234, ISSN 0013-4686.

Tang, G. \& Bai, N. (2000). An analysis of precipitation chemistry data in China with genetic neural network. Acta Scientiae Circumstance, 20, 542-, ISSN 0253-2468.

Vinnichenko, M.; Pham, M. \& Chevolleau, T.; et al. (2003). In situ ellipsometric investigation of stainless steel corrosion behavior in buffered solutions with amino acids. Applied Surface Science, 207, 176-182, ISSN 0169-4332.

Wei, Y. \& Xu, B. (2007). Theory and practice of corrosion and protection for magnesium alloys. Metallurgy Industry Press of China, ISBN 978-7-5024-4232-3, Beijing.

Zhang, J. \& Zhang, Z. (2004). Magnesium alloys and their applications, Chemical Industry Press of China, ISBN 7-5025-5709-9, Beijing. 


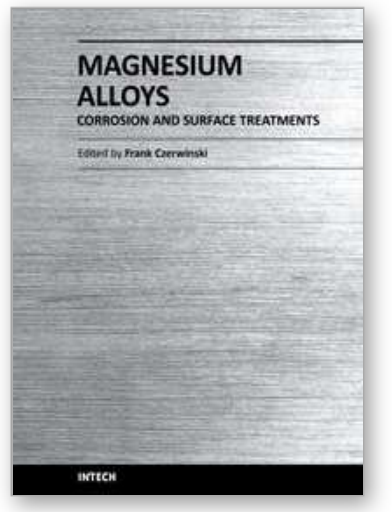

\author{
Magnesium Alloys - Corrosion and Surface Treatments \\ Edited by Frank Czerwinski
}

ISBN 978-953-307-972-1

Hard cover, 344 pages

Publisher InTech

Published online 14, January, 2011

Published in print edition January, 2011

A resistance of magnesium alloys to surface degradation is paramount for their applications in automotive, aerospace, consumer electronics and general-purpose markets. An emphasis of this book is on oxidation, corrosion and surface modifications, designed to enhance the alloy surface stability. It covers a nature of oxides grown at elevated temperatures and oxidation characteristics of selected alloys along with elements of general and electrochemical corrosion. Medical applications are considered that explore bio-compatibility of magnesium alloys. Also techniques of surface modifications, designed to improve not only corrosion resistance but also corrosion fatigue, wear and other behaviors, are described. The book represents a valuable resource for scientists and engineers from academia and industry.

\title{
How to reference
}

In order to correctly reference this scholarly work, feel free to copy and paste the following:

Jinglei Lei, Fusheng Pan and Lingjie Li (2011). In Situ Ellipsometric Study on Corrosion of Magnesium Alloys, Magnesium Alloys - Corrosion and Surface Treatments, Frank Czerwinski (Ed.), ISBN: 978-953-307-972-1, InTech, Available from: http://www.intechopen.com/books/magnesium-alloys-corrosion-and-surfacetreatments/in-situ-ellipsometric-study-on-corrosion-of-magnesium-alloys

\section{INTECH}

open science | open minds

\section{InTech Europe}

University Campus STeP Ri

Slavka Krautzeka 83/A

51000 Rijeka, Croatia

Phone: +385 (51) 770447

Fax: +385 (51) 686166

www.intechopen.com

\section{InTech China}

Unit 405, Office Block, Hotel Equatorial Shanghai

No.65, Yan An Road (West), Shanghai, 200040, China

中国上海市延安西路65号上海国际贵都大饭店办公楼 405 单元

Phone: +86-21-62489820

Fax: $+86-21-62489821$ 
(C) 2011 The Author(s). Licensee IntechOpen. This chapter is distributed under the terms of the Creative Commons Attribution-NonCommercialShareAlike-3.0 License, which permits use, distribution and reproduction for non-commercial purposes, provided the original is properly cited and derivative works building on this content are distributed under the same license. 